\title{
Effects of the Third-Order Interaction of Polymer Chains on Average Bond Conformations
}

\author{
Linxi ZHANG \\ Department of Physics, Hangzhou University, \\ Hangzhou, 310028, People's Republic of China
}

(Received October 26, 1992)

\begin{abstract}
The a priori probabilities of polymer chains having a one-bond repeat unit and a two-bond repeat unit in the third-order interaction approximation are obtained. The probability may be as a tool to assess the energies of various rotational isomeric stages. Comparisons with the second-order interaction calculations are also made.

KEY WORDS A Priori Probability / Third-Order Interaction / Second-Order

Interaction /
\end{abstract}

Solution properties of polymers depend strongly on the conformational behavior of polymer chains. These properties must be averaged over all conformation of a chain, in which there are a large number of interactions between atoms. Accurate calculation of these interactions are however difficult, and can be done only approximately. Configurational statistics of unperturbed polymer chain calculated by the matrix algebra method, mainly considering the second-order interactions between pairs of atoms separated by four bonds. ${ }^{1-6}$ Owing to the fact that no particular conformation or restricted set of conformations is decisively preferred as a result of interactions of first and second orders, those of third and fourth orders may be important. ${ }^{7}$ The third or higher order interactions may be important for the temperature coefficient $\mathrm{d} \ln \left\langle R^{2}\right\rangle / \mathrm{d} T$ of polydimethylsiloxane (PDMS). ${ }^{8}$

Recently Jianmin et al. ${ }^{9}$ have performed calculation including the third-order interaction imposed by pairs of atoms separated by five bonds. $\mathrm{We}^{10}$ have also studied the unperturbed dimensions and dipole moments of polydimethylsilmethylene (PDMSM), and found the characteristic retios of $\left\langle R^{2}\right\rangle_{0}$ and $\left\langle\mu^{2}\right\rangle_{0}$ at $30^{\circ} \mathrm{C}$ in the third-order interaction are 5.4 and 0.34 , respectively, and these results are in satisfactory agreement with the experimental data, 5.32 and 0.39 , respectively. ${ }^{11}$ However, they are 4.1 and 0.23 in the second-order interaction respectively and 3.8 and 0.21 in the first-order interaction, respectively. ${ }^{10}$ The unperturbed dimension of PDMSM chain becomes large. The characteristic ratio of $\left\langle R^{2}\right\rangle_{0}$ in the third-order interaction is larger than that in the second-order interaction by $32 \%$, and than that in the first-order interaction by $42 \%$. For some chains, the second-order interaction can be well enough in explaining their conformational properties, but for those chains of more flexibility, it may be important to carry out calculations based on the third-order interaction or furthermore, fourth-order interaction. For example, the third-order interaction is important for PDMSM chain and the fourthorder interaction is important for POM(polyoxymethylene) chain. ${ }^{12}$ The investigation of the higher order interaction may provide some insight into the short-range interaction of polymer chains. In the rotational isomeric-state model of polymer chains, each skeletal bond 
is assigned to one of a small number of discrete, rotational states. The probability of rotational state is different and depends on the interactions between atoms. Therefore, the probabilities of rotational states may provide some insight into the bond conformations of polymer chains. The probability may be as a tool to assess the energies of various rotational isomeric states. ${ }^{8}$ In this paper, the effects of the third-order interactions on average bond conformations are investigated.

\section{METHODS OF CALCULATION}

The a priori probabilities $p_{\xi \eta}$ of $\xi \eta$ states are defined by

$$
p_{\xi \eta}=(n-3)^{-1} \sum_{i=3}^{n-1} p_{\xi \eta, i}
$$

where $p_{\xi \eta, i}$ is the probability that bonds $i-1$ and $i$ occur simultaneously in states $\xi$ and $\eta$, respectively. The a priori probability $p_{\xi \eta}$ is also the average fraction of the $n-3$ relevant bond pairs in paired states,

$$
p_{\xi \eta}=\left\langle n_{\xi \eta}\right\rangle /(n-3)
$$

where $\left\langle n_{\xi \eta}\right\rangle$ is the average number of bond pairs in states $\xi \eta$, and given by

$$
\left\langle n_{\xi \eta}\right\rangle=Z^{-1} \sum n_{\xi \eta} \Omega_{\{\phi\}}
$$

where $n_{\xi \eta}$ is the number of $\xi \eta$ pairs in configuration $\Omega$, therefore the a priori probabilities $p_{\xi \eta}$ are given by

$$
p_{\xi \eta}=(n-3)^{-1}\left(\partial \ln Z / \partial \ln \mu_{\xi \eta}\right)
$$

For large $n$,

$$
p_{\xi \eta}=\partial \ln \lambda_{1} / \partial \ln \mu_{\xi \eta}
$$

where $\lambda_{1}$ is the largest eigenvalue of matrix $\boldsymbol{U}$.

\section{PM Chain}

Polymethylene (PM) chain represents the simplest case of a chain having a one-bond repeat unit. The a priori probabilities of PM chains in the second-order interaction approximation are given by ${ }^{1}$

$$
p_{\xi \eta}=\mu_{\xi \eta} A_{\eta 1} B_{1 \zeta} / \lambda_{1}, \quad n \rightarrow \infty
$$

where $A_{\eta 1}$ and $B_{1 \xi}$ are elements of the eigenvector and eigenrow matrices $\boldsymbol{A}$ and $\boldsymbol{B}$ of the statistical weight matrix $\boldsymbol{U}$, and $\boldsymbol{B}=\boldsymbol{A}^{-1}$.

The $a$ priori probability of state $\eta$ for a long chain is

$$
p_{\eta}=A_{\eta 1} B_{1 \eta}, \quad n \rightarrow \infty
$$

After a derivation similar to the second-order interaction, the a priori probabilities of PM chains in the third-order interaction are given by

$$
p_{\xi \eta \zeta}^{\prime}=\mu_{\alpha \beta}^{\prime} A_{\beta 1}^{\prime} B_{1 \alpha}^{\prime} / \lambda_{1}^{\prime}, \quad n \rightarrow \infty
$$

where $\mu_{\alpha \beta}^{\prime}$ is the element of the third-order interaction statistical weight matrices $U^{\prime}$. $A_{\beta 1}^{\prime}$ and $B_{1 \alpha}^{\prime}$ are elements of the eigenvector and eigenrow matrices $\boldsymbol{A}^{\prime}$ and $\boldsymbol{B}^{\prime}$ of matrix $\boldsymbol{U}^{\prime} . \alpha$ is $1,2, \cdots, 9$, and responds to $t t, t^{+}, t g^{-}, g^{+} t$, $g^{+} g^{+}, g^{+} g^{-}, g^{-} t, g^{-} g^{+}$, and $g^{-} g^{-}$, respectively. $\beta$ is $1,2, \cdots, 9$, and responds to $t, g^{+}$, $g^{-}, t, g^{+}, g^{-}, t, g^{+}$, and $g^{-}$, respectively. The value of $\alpha$ depends on the states $\xi \eta$, and the value of $\beta$ depends on the state $\zeta$ and matrix $\boldsymbol{U}^{\prime}$. For example, the values of $\alpha$ and $\beta$ for $g^{+} g^{+} t$ states are 5 and 4 , respectively.

\section{PDMSM Chain}

The polydimethylsilmethylene (PDMSM) chain is the case of a chain having a two-bond repeat unit. In the second-order interaction approximation, two statistical weight matrices are requied for a description of its configurations, one for the pair of bonds $\mathrm{Si}-\mathrm{C}$ and $\mathrm{C}-\mathrm{Si}$ flanking a $\mathrm{C}$ atom and the other for the pair $\mathrm{C}-\mathrm{Si}$ and $\mathrm{Si}-\mathrm{C}$ flanking a $\mathrm{Si}$ atom. The matrices of PDMSM chain are, respectively,

$$
\begin{array}{r}
\boldsymbol{U}_{a}=\left[\begin{array}{ccc}
1 & \sigma & \sigma \\
1 & \sigma \psi_{a} & \sigma \omega_{a} \\
1 & \sigma \omega_{a} & \sigma \psi_{a}
\end{array}\right] \\
\boldsymbol{U}_{b}=\left[\begin{array}{ccc}
1 & \sigma & \sigma \\
1 & \sigma \psi_{b} & \sigma \omega_{b} \\
1 & \sigma \omega_{b} & \sigma \psi_{b}
\end{array}\right]
\end{array}
$$


the a priori probabilities of PDMSM chain for bond pair $a$ are

$$
\begin{aligned}
& p_{g^{+} g^{+, a}}=\left(\psi_{a} / 2 \lambda_{1}\right)\left(\partial \lambda_{1} / \partial \alpha\right) \\
& p_{g^{+} g^{-}, a}=\left(\omega_{a} / 2 \lambda_{1}\right)\left(\alpha \lambda_{1} / \partial \alpha\right)
\end{aligned}
$$

where

$$
\begin{aligned}
& \boldsymbol{U}=\boldsymbol{U}_{\boldsymbol{a}} \cdot \boldsymbol{U}_{b} \\
& \alpha=\psi_{a}+\omega_{a} \\
& \beta=\psi_{b}+\omega_{b}
\end{aligned}
$$

$\lambda_{1}$ is the largest eigenvalue of matrix $\boldsymbol{U}$. All of the $a$ priori probabilities can be deduced from the three partial derivatives $\partial \lambda_{1} / \partial \sigma, \partial \lambda_{1} / \partial \alpha$, and $\partial \lambda_{1} / \partial \beta$.

In the third-order interaction approximation, because the statistical weight matrices for $\mathrm{Si}-\mathrm{CH}_{2}-\mathrm{Si}-\mathrm{CH}_{2}-\mathrm{Si}-\mathrm{CH}_{2}$ bond pairs and for $\mathrm{CH}_{2}-\mathrm{Si}-\mathrm{CH}_{2}-\mathrm{Si}-\mathrm{CH}_{2}-\mathrm{Si}$ bond pairs are the same, the a priori probabilities of PDMSM chain are also given by eq 8 . Theoretically, the method of calculation the a priori probability in the higher order interaction are the same. It does not need to go up to the higher order for PDMSM chain because of the difference between the third-order and the higher order are insignificant.

\section{RESULTS AND DISCUSSION}

The statistical weight matrix of PM chain in the second-order interaction is

$$
\boldsymbol{U}=\left[\begin{array}{ccc}
1 & \sigma & \sigma \\
1 & \sigma & \sigma \omega \\
1 & \sigma \omega & \sigma
\end{array}\right]
$$

In the third-order interaction approximation, the statistical weight matrix is ${ }^{9}$

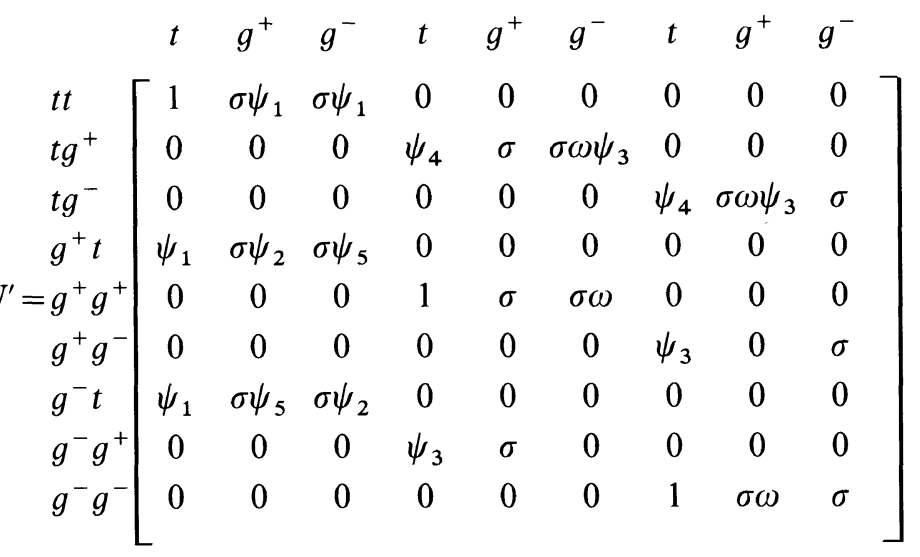

where

$$
\begin{aligned}
\sigma & =\exp \left(-E_{\sigma} / R T\right) \\
\omega & =\exp \left(-E_{\omega} / R T\right) \\
\psi_{i} & =\exp \left(-E \psi_{i} / R T\right)
\end{aligned}
$$

$g^{ \pm} g^{\mp}$ ) and $E_{\psi_{i}}$ the third-order. They are computed with the semiempirical formula

$$
\begin{aligned}
E= & \left(E_{0} / 2\right)\left(1-\cos 3 \phi_{i}\right) \\
& +\sum_{k<1}\left(a_{k 1} \exp \left(-b_{k 1} r_{k 1}\right)-c_{k 1} / r_{k 1}^{6}\right)
\end{aligned}
$$

Values of the various parameters adopted for use in eq 17 refer to ref 2 . At $140^{\circ} \mathrm{C}$, they are 


$$
\left.\begin{array}{r}
\sigma=0.524 \\
\omega=0.087 \\
\psi_{1}=1.102 \\
\psi_{2}=1.356 \\
\psi_{3}=1.261 \\
\psi_{4}=1.143 \\
\psi_{5}=1.063
\end{array}\right\}
$$

The third-order interaction energy $\left|E_{\psi_{i}}\right|\left(E_{\psi_{i}}<\right.$ $0)$ is small. For example, the largest ratio of $\left|E_{\psi_{i}}\right| / E_{\sigma}$ is 0.471 .

The a priori probabilities in the second-order interaction, calculated by eq 6 and expressed for convenience in the form of a matrix, are

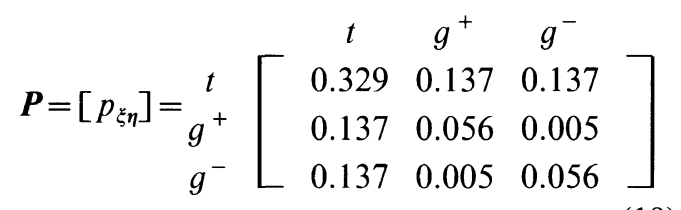

The a priori probability of state $\eta$, calculated by eq 7 , is

$$
p_{t}=0.603, \quad p_{g^{+}}=p_{g^{-}}=0.198
$$

In the third-order interaction approximation, the largest eigenvalue of matrix $U^{\prime}$ is 1.99 . The required eigenvector and eigenrow matrices $\boldsymbol{A}_{1}^{\prime}$ and $\boldsymbol{B}_{1}^{\prime}$ are

$$
\begin{aligned}
& A_{1}^{\prime}=\left[A_{\beta 1}^{\prime}\right]=\left[\begin{array}{l}
0.582 \\
0.500 \\
0.500 \\
0.641 \\
0.454 \\
0.526 \\
0.641 \\
0.525 \\
0.218
\end{array}\right]
\end{aligned}
$$

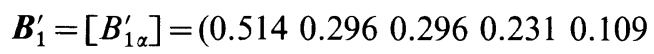

$$
\begin{aligned}
& \left.\begin{array}{lllllll}
0.009 & 0.231 & 0.009 & 0.109
\end{array}\right)
\end{aligned}
$$

Thus, the a priori probabilities for large $n$, caluculated by eq 8. are

$$
\begin{aligned}
& \begin{array}{l}
t \quad g^{+} \\
g^{-}
\end{array}
\end{aligned}
$$

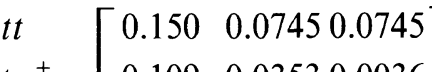

$$
\begin{aligned}
& \mathrm{tg}^{+} \quad 0.109 \quad 0.03530 .0036 \\
& \begin{array}{llll}
\mathrm{tg}^{-} & 0.109 & 0.0036 & 0.0353
\end{array} \\
& g^{+} t \quad 0.07450 .04120 .0323 \\
& \boldsymbol{P}^{\prime}=\left[\begin{array}{llllll}
P_{\xi \eta \xi}^{\prime}
\end{array}\right]=g^{+} g^{+} \quad \begin{array}{llll}
0.0351 & 0.0130 & 0.0013 & (22)
\end{array} \\
& \begin{array}{l|lll}
g^{+} g^{-} & 0.0038 & 0 & 0.0005
\end{array} \\
& \begin{array}{l|l}
g^{-} t & 0.07450 .03230 .0412
\end{array} \\
& g^{-} g^{+} \quad 0.00380 .0005 \quad 0 \\
& g^{-} g^{-}[0.03510 .00130 .0130
\end{aligned}
$$

In the third-order interaction, the probability of $t t t$ pairs is 0.150 , and the largest one. The $g^{-} g^{\mp} g^{ \pm}$conformation energies are in excess of $5.4 \mathrm{kcal} \mathrm{mol}^{-1}$, which are so high as to be impossible to generate, ${ }^{9}$ therefore the probabilities are 0 . We also calculate the a priori probabilities $p_{\xi \eta}^{\prime \prime}$, i.e., $p_{\xi \eta}^{\prime \prime}=\sum_{\zeta} p_{\xi \eta \zeta}^{\prime}$, of $\xi \eta$ pairs in the third-order interaction, and given by

$$
\begin{aligned}
& \boldsymbol{P}^{\prime \prime}=\left[p_{\xi \eta}^{\prime \prime}\right]= \\
& g^{+} g^{-}\left[\begin{array}{ccc}
t & g^{+} & g^{-} \\
0.299 & 0.148 & 0.148 \\
0.148 & 0.0494 & 0.0043 \\
0.148 & 0.0043 & 0.0494
\end{array}\right]
\end{aligned}
$$

In the third-order interaction, the probability of $t t$ pairs is $p_{t t}^{\prime}=0.299$, and less than that in the second-order. The probabilities of $t g^{ \pm}$, $g^{ \pm} t$ pairs are $p_{g^{ \pm} t}^{\prime \prime}=p_{t g^{ \pm}}^{\prime \prime}=0.148$, and greater than those in the second-order. The probability of state $t p_{t}^{\prime \prime}=0.595$, and less than that in the second-order. Thus, the difference between the second-order and the third-order for PM chain is small. This means the higher order interactions are not important for PM chain.

The statistical weight matrices of PDMSM chain in the second-order interaction approximation are $^{10,11}$ 


$$
\begin{aligned}
& U_{a}=\left[\begin{array}{lll}
1 & 1 & 1 \\
1 & 1 & 1 \\
1 & 1 & 1
\end{array}\right] \\
& U_{b}=\left[\begin{array}{lll}
1 & 1 & 1 \\
1 & 1 & \omega \\
1 & \omega & 1
\end{array}\right]
\end{aligned}
$$

In the third-order interaction, the statistical weight matrices of PDMSM are given ${ }^{10}$

$\boldsymbol{U}_{a}^{\prime}=\boldsymbol{U}_{b}^{\prime}=$

$$
\left[\begin{array}{ccccccccc}
1 & \psi_{1} & \psi_{1} & 0 & 0 & 0 & 0 & 0 & 0 \\
0 & 0 & 0 & \psi_{2} & 1 & 0 & 0 & 0 & 0 \\
0 & 0 & 0 & 0 & 0 & 0 & \psi_{2} & 0 & 1 \\
1 & \psi_{3} & \psi_{4} & 0 & 0 & 0 & 0 & 0 & 0 \\
0 & 0 & 0 & 1 & \psi_{5} & 0 & 0 & 0 & 0 \\
0 & 0 & 0 & 0 & 0 & 0 & 0 & 0 & \psi_{6} \\
1 & \psi_{4} & \psi_{3} & 0 & 0 & 0 & 0 & 0 & 0 \\
0 & 0 & 0 & 0 & \psi_{6} & 0 & 0 & 0 & 0 \\
0 & 0 & 0 & 0 & 0 & 0 & 1 & 0 & \psi_{5}
\end{array}\right]
$$

where

$$
\begin{gathered}
\omega=\exp \left(-E_{\omega} / R T\right) \\
\psi_{i}=\exp \left(-E_{\psi_{i}} / R T\right)
\end{gathered}
$$

The conformation energies $E_{\omega}$ and $E_{\psi_{i}}$ are computed with the potential function

$$
\begin{aligned}
E= & \left(E_{0} / 2\right)\left(1-\cos 3 \phi_{i}\right)+\sum_{k<l}\left(\left(a_{k l} / r_{k l}^{12}-b_{k l} / r_{k l}^{6}\right)\right. \\
& \left.+332.2 \cdot \delta_{k} \delta_{l} / r_{k l}\right)
\end{aligned}
$$

Values of various parameters refer to ref 13 . At $30^{\circ} \mathrm{C}$, the statistical weight factors are

$$
\left.\begin{array}{l}
\omega=0.833 \\
\psi_{1}=0.572 \\
\psi_{2}=0.468 \\
\psi_{3}=0.651 \\
\psi_{4}=0.536 \\
\psi_{5}=0.897 \\
\psi_{6}=0.464
\end{array}\right\}
$$

$\omega$ is the second-order statistical weight factor and $\psi_{i}(i=1, \cdots, 6)$ are the third-order statistical weight factors. $E_{\psi_{i}}$ are the extra nonbonded interaction energies and Coulombic interaction energies of the sixth skeletal atom. The larger the extra energies are, the more the difference between the second-order and the third-order is. The third-order interaction depends on the chain structure. If $E_{\psi_{i}}(i=1, \cdots, 6)$ are close to 0 , the third-order interaction may be neglected. However, $E_{\psi_{2}}$ of PDMSM chain is large enough, for example, the largest ratio of $E_{\psi_{i}} / E_{\sigma}$ is 4.2 , therefore the

\begin{tabular}{|c|c|}
\hline & $g^{+}$ \\
\hline$t t$ & $0.106 \quad 0.08050 .0805^{-}$ \\
\hline $\operatorname{tg}^{+}$ & 0.07930 .0723 \\
\hline $\operatorname{tg}^{-}$ & $\begin{array}{lll}0.0793 & 0 & 0.0723\end{array}$ \\
\hline$g^{+} t$ & 0.07520 .04190 .0342 \\
\hline $\boldsymbol{P}_{a}^{\prime \prime}=\boldsymbol{P}_{b}^{\prime \prime}=g^{+} g^{+}$ & 0.03870 .0245 \\
\hline$g^{+} g^{-}$ & 0.0005 \\
\hline$g^{-} t$ & 0.07520 .03420 .0419 \\
\hline$g^{-} g^{+}$ & 0.0005 \\
\hline$g^{-} g^{-}$ & $\begin{array}{lll}0.0387 & 0 & 0.0245\end{array}$ \\
\hline
\end{tabular}
third-order interaction should be considered.

The a priori probabilities in the second-order, expressed for convenience in the form of matrices, are ${ }^{1}$

$$
\begin{aligned}
& \boldsymbol{P}_{a}=\left[\begin{array}{lll}
0.111 & 0.111 & 0.111 \\
0.111 & 0.111 & 0.111 \\
0.111 & 0.111 & 0.111
\end{array}\right] \\
& \boldsymbol{P}_{b}=\left[\begin{array}{lll}
0.080 & 0.120 & 0.120 \\
0.120 & 0.120 & 0.100 \\
0.120 & 0.100 & 0.120
\end{array}\right]
\end{aligned}
$$

Thus, the a priori probabilities of state $\eta$ are

$$
\begin{aligned}
& p_{a, t}=p_{a, g^{+}}=p_{a, g^{-}}=0.333, \\
& p_{b, t}=0.320, \quad p_{b, g^{+}}=p_{b, g^{-}}=0.340
\end{aligned}
$$

Similar to PM chain, the third-order a priori probabilities of PDMSM chain, calculated by eq 8 , are 
The probability of $t t t$ pairs of PDMSM chain is the largest one. In the third-order interaction, the $g^{ \pm} g^{ \pm} g^{\mp}, g^{ \pm} g^{\mp} g^{ \pm}, g^{ \pm} g^{\mp} t$, and $t g^{ \pm} g^{\mp}$ conformations are impossible to generate, and the probabilities are 0 . The a priori probabilities $P_{\xi \eta}^{\prime \prime}$ of $\xi_{\eta}$ pairs in the third-order interaction are

$$
\boldsymbol{P}_{a}^{\prime \prime}=\boldsymbol{P}_{b}^{\prime \prime}=\left[\begin{array}{lll}
0.267 & 0.151 & 0.151 \\
0.151 & 0.063 & 0.0005 \\
0.151 & 0.0005 & 0.063
\end{array}\right]
$$

The probabilities of $g^{ \pm} g^{ \pm}$and $g^{ \pm} g^{\mp}$ pairs are less than those in the second-order, and the probability of $t$ state $p_{t}^{\prime \prime \prime}=0.570$ is greater than that in the second-order by $40 \%$. It is evident that the chain are extended and the dimension of PDMSM chain become large. Thus, the third-order interaction are important for PDMSM chain. The a priori probabilities of pairs can explain the reason the dimensions of polymer chains become large or small. The statistical weight matrices are usually estimated from conformational energy calculations for small molecules. Recently, the new approach, ${ }^{8}$ which is via the analysis of a molecular dynamics trajectory that samples all conformational space for a fragment of the polymer, has been adopted in the applications of rotational isomeric-state theory. Configuration-dependent properties of some polymer chains can be assessed by calculation of the third-order probabilities extracted from the molecular dynamics trajectory.

Acknowledgment. This work was supported by Natural Science Function of Zhejiang Province of China. The author would like to thank Professor Xu Jianmin for helpful discussions.

\section{REFERENCES}

1. P. J. Flory, "Statistical Mechanics of Chain Molecules," Interscience Publishers, New York, 1969.

2. A. Abe, R. L. Jernigan, and P. J. Flory, J. Am. Chem. Soc., 88, 631 (1966).

3. D. Y. Yoon, P. R. Sundararajan, and P. J. Flory, Macromolecules, 8, 776 and 784 (1975).

4. P. J. Flory and D. Y. Yoon, J. Chem. Phys., 61, 5358 (1974).

5. D. Y. Yoon and P. J. Flory, J. Chem. Phys., 61, 5366 (1974).

6. T. M. Birshtein and D. B. Ptitsyn, "Conformation of Macromolecules," Interscience Publishers, New York, 1966.

7. P. J. Flory, P. R. Sundararajan, and L. C. DoBolt, J. Am. Chem. Soc., 96, 5015 (1974).

8. I. Bahar, I. Zuniga, R. Dodge, and W. L. Mattice, Macromolecules, 24, 2993 (1992).

9. X. Jianmin, S. Xubing, Z. Zhiping, and Y. Deyue, J. Polym. Sci., Polym. Phys. Ed., 29, 877 (1991).

10. W. Xiaoyi, J. Jinsheng, Z. Linxi, and X. Jianmin, $J$. Polym. Sci., Polym. Phys. Ed., 31, 455 (1993).

11. J. K. Ko and J. E. Mark, Macromolecules, 8, 869 (1975).

12. J. Tao, J. Jinsheng, Z. Linxi, and X. Jianmin, submitted for publication in Eur. Polym. J.

13. S. Brucker and L. Malpezzi, Makromol. Chem., 183, 2033 (1982). 